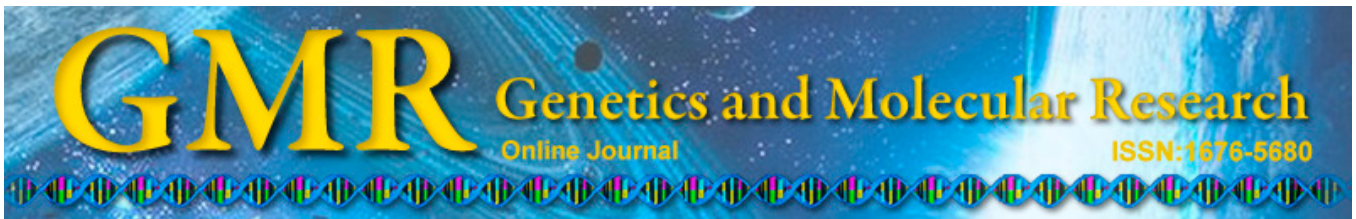

\title{
CYP2E1 PstI polymorphism increases cervical neoplasia risk: a meta-analysis
}

\author{
X. Wang and Y. He \\ Department of Gynecology, West China Second University Hospital, \\ Sichuan University, Chengdu, Sichuan, China \\ Corresponding author: Y. He \\ E-mail: hydscu@163.com
}

Genet. Mol. Res. 14 (2): 5203-5209 (2015)

Received July 22, 2014

Accepted December 3, 2014

Published May 18, 2015

DOI http://dx.doi.org/10.4238/2015.May.18.11

\begin{abstract}
Cytochrome P4502E1 (CYP2E1) is a key enzyme in the metabolic activation of many carcinogens, but the roles of CYP2E1 polymorphisms in cervical neoplasia $(\mathrm{CN})$ are inconclusive. Published case-control cohort studies from the Pubmed, Embase, and China National Knowledge Infrastructure databases were retrieved. Data were extracted and pooled odds ratios with 95\% confidence intervals were calculated. Seven studies examining 1097 cases and 1117 controls were included in this meta-analysis. The pooled effect size showed no association between $C Y P 2 E 1$ RsaI and DraI polymorphisms and CN risk in a codominant model. However, using a recessive model, an association between the Pst $\mathrm{I}$ polymorphism and $\mathrm{CN}$ risk was observed (odds ratio: $2.10,95 \%$ confidence interval: 0.96-4.62, $\mathrm{P}=0.06$ ), indicating that individuals with the homozygous rare genotype have a higher risk of developing $\mathrm{CN}$ compared to those with homozygous wildtype and heterozygous genotypes. When stratified by ethnicity, the Pst polymorphism was significantly correlated with $\mathrm{CN}$ susceptibility in non-Asians (odds ratio: $3.74,95 \%$ confidence interval: $1.13-12.43, \mathrm{P}=$
\end{abstract}


0.03). This meta-analysis suggests that the CYP2E1 PstI polymorphism increases the risk of $\mathrm{CN}$ in non-Asians.

Key words: Cervical neoplasia; Cytochrome P450; Polymorphism

\section{INTRODUCTION}

Cytochrome P4502E1 (CYP2E1) is a member of the cytochrome P450 superfamily and a key enzyme in the metabolic activation of many carcinogens. The DraI, PstI, and RsaI restriction fragment length polymorphism patterns of the CYP2E1 gene are associated with a predisposition to cancer in humans (Agundez, 2004).

Cervical cancer is the second most common type of cancer among women worldwide. In addition to other risk factors (human papilloma virus infections, chemical reagents exposure, etc.), genetic background independently plays an important role in this process $(\mathrm{Au}$ et al., 2003). Over the past decade, several studies have focused on the relationship between CYP2E1 gene polymorphisms and cervical neoplasia $(\mathrm{CN})$, including high-grade squamous intraepithelial lesion, cervical intraepithelial neoplasia, carcinoma in situ, and invasive cervical cancer (Kim et al., 2000; Ferreira et al., 2006; Sierra-Torres et al., 2003, 2006; Nishino et al., 2008; Liu et al., 2009; von Keyserling et al., 2011). However, the results were not conclusive. In this study, we performed a meta-analysis to determine the relationship between CYP2E1 gene polymorphisms and $\mathrm{CN}$ risk.

\section{MATERIAL AND METHODS}

\section{Search strategy}

A literature search was conducted using the Pubmed, Embase, and China National Knowledge Infrastructure (www.cnki.net) databases. The following search terms were utilized: cytochrome P4502E1 or CYP2E1, and gene polymorphism or polymorphism or variant, and cervical squamous intraepithelial lesion or cervical intraepithelial neoplasia or cervical neoplasia or cervical carcinoma or cervical cancer.

\section{Data extraction}

Two independent reviewers collected the data according to inclusion and exclusion criteria. For inclusion in the meta-analysis, retrieved articles had to state the number of cases and controls and number of individual genotypes in cases and controls. Exclusion criteria in the meta-analysis were: 1) not a genetic study, 2) duplicated report, and 3) no useful data reported. Unpublished data were not considered. Disagreement was resolved by discussion before reaching a consensus.

\section{Statistical analyses}

Categorical variables were presented as the odds ratio (OR) with $95 \%$ confidence interval (CI). For CYP2E1 DraI, PstI, and RsaI polymorphisms, the 3 different genotypes were referred to as the homozygous wild-type genotype (c1c1), heterozygous genotype (c1c2), and 
homozygous rare genotype (c2c2), respectively. OR1, OR2, and OR3 were calculated as follows: 1) c2c2 vs c1c1; 2) c1c2 vs c1c1; 3) c2c2 vs c1c2. Pairwise differences (OR1, OR2, and OR3) were used to identify the most appropriate genetic model as previously described (Chen et al., 2013). Pooled ORs with $95 \%$ CIs were calculated and $\mathrm{P}<0.05$ was considered to be statistically significant. Heterogeneity was evaluated using the $Q$ test. Meta-analysis was conducted using the fixed-effect model when there was no heterogeneity $(\mathrm{P} \geq 0.1)$. Otherwise, the random-effect model was used. Subgroup analysis was performed by ethnicity. The Begg rank correlation test and the Egger linear regression test were used to identify potential publication bias. All analyses were conducted using Revman 5.0 (Cochrane Collaboration, Oxford, UK) and Stata 11.0 (StataCorp, College Station, TX, USA).

\section{RESULTS}

\section{Studies included in the meta-analysis}

Forty-two studies were relevant to the search terms. After reviewing the titles, abstracts, and articles, 35 studies were excluded, and 7 studies examining 1097 cases and 1117 controls matched the inclusion criteria (Figure 1). Of the 7 included studies, 6 were published in English and 1 was published in Chinese. These studies were carried out in China, Japan, Korea, USA, Portugal, Colombia, and Germany. Although the ethnicity was not indicated by Sierra-Torres et al. (2006), a non-Asian mixed population in Colombia was assumed. The main features of the included studies are presented in Table 1.

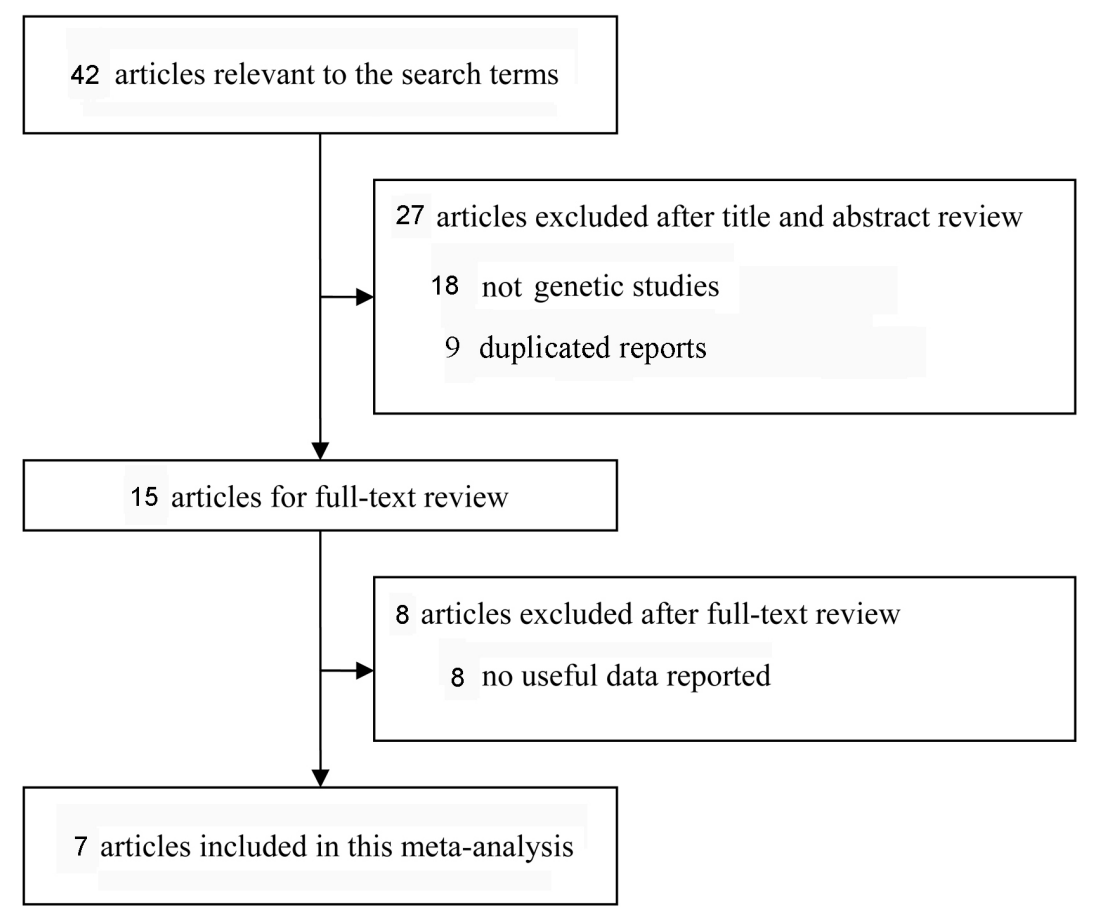

Figure 1. Flow diagram of search process. 


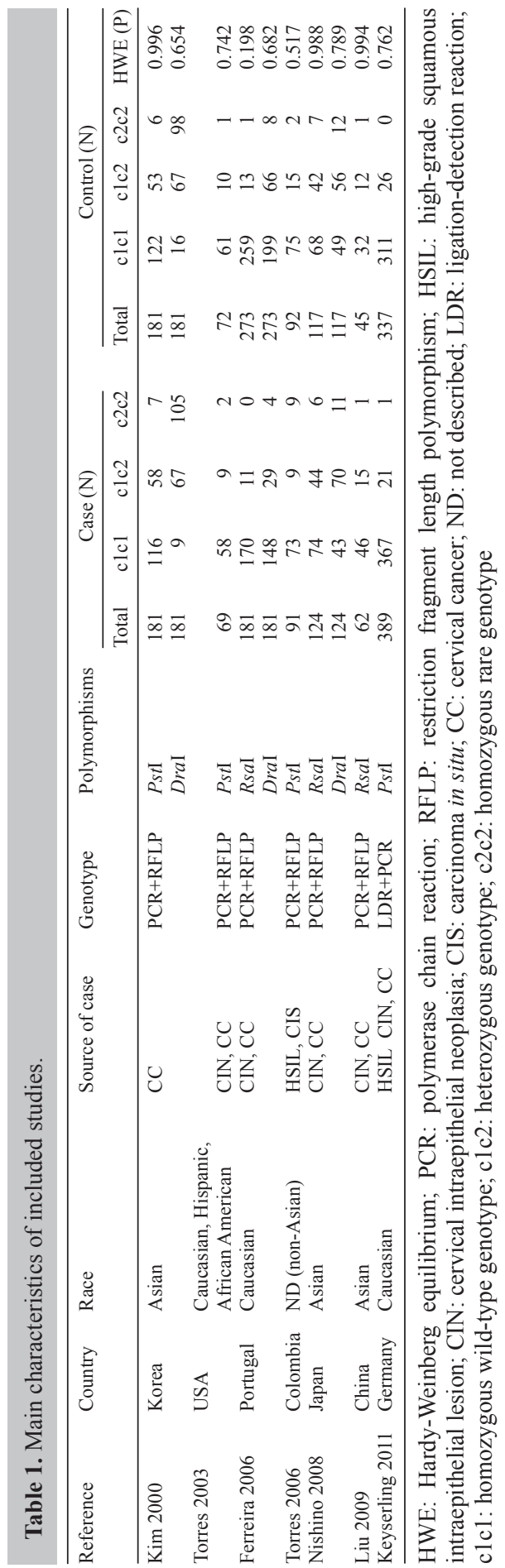




\section{Quantitative synthesis}

After calculating OR1, OR2, and OR3, the most appropriate genetic models for the $P s t \mathrm{I}, R s a \mathrm{I}$, and DraI polymorphisms were the recessive model, codominant model, and codominant model, respectively. In the codominant model, no association was observed between the RsaI and DraI polymorphisms and CN risk (data not shown). However, using the recessive model, the pooled effect size showed an association between the Pst $\mathrm{I}$ polymorphism and $\mathrm{CN}$ risk (c2c2 vs $\mathrm{c} 1 \mathrm{c} 1+\mathrm{c} 1 \mathrm{c} 2$, OR: $2.10,95 \% \mathrm{CI}$ : 0.96-4.62, $\mathrm{P}=0.06$, Figure 2), indicating that individuals with the homozygous rare genotype had a higher, but not significant, risk of CN compared to those with the homozygous wild-type genotype and heterozygous genotype. In subgroup analysis by ethnicity, the results indicated that the PstI polymorphism was significantly correlated with $\mathrm{CN}$ susceptibility in non-Asians (c2 2 vs $\mathrm{c} 1 \mathrm{c} 1+\mathrm{c} 1 \mathrm{c} 2$, OR: 3.74, 95\%CI: $1.13-12.43, \mathrm{P}=0.03$, Figure 2).

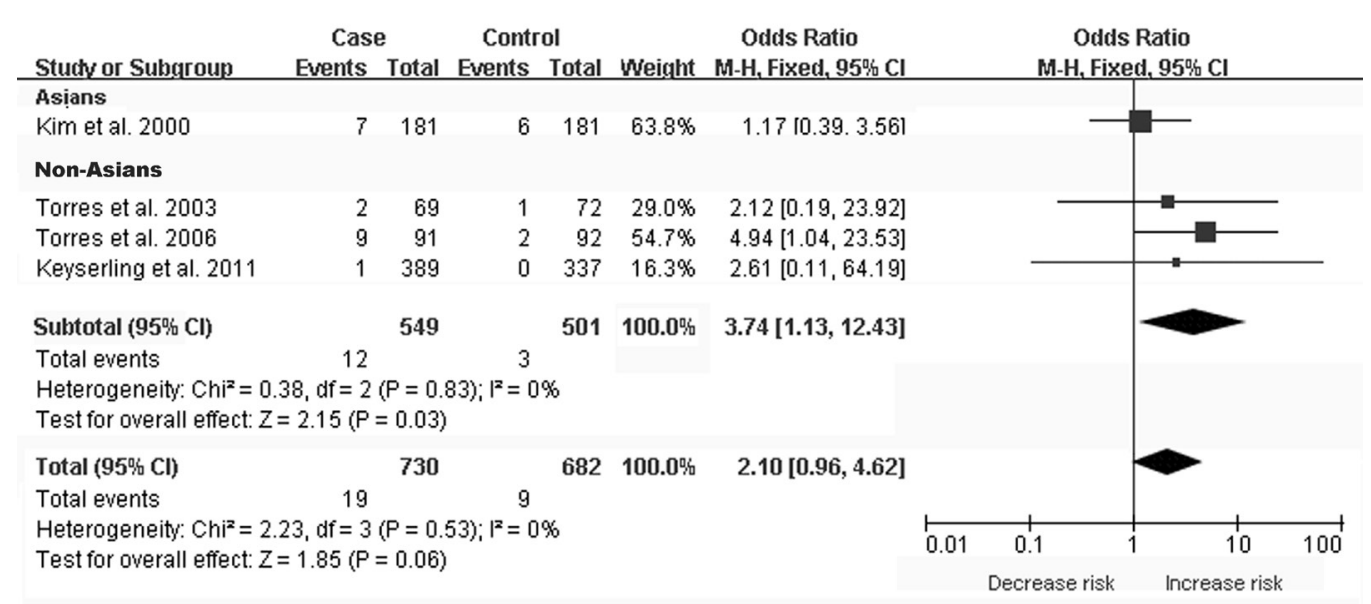

Figure 2. Forest plots of OR with $95 \% \mathrm{CI}$ for the association between CYP2E1 PstI polymorphism and cervical neoplasia risk $(\mathrm{c} 2 \mathrm{c} 2$ vs $\mathrm{c} 1 \mathrm{c} 1+\mathrm{c} 1 \mathrm{c} 2)$.

\section{Analyses of heterogeneity, sensitivity, and publication bias}

Significant heterogeneity was not observed between all studies in $\mathrm{c} 2 \mathrm{c} 2 v s \mathrm{c} 1 \mathrm{c} 1+\mathrm{c} 1 \mathrm{c} 2$ for the PstI polymorphism. When stratified by ethnicity, no heterogeneity was observed in non-Asians. After exclusion of each study, the pattern of the pooled effect size was still significant for the PstI polymorphism. Publication bias was not observed based on the Begg rank correlation test $(\mathrm{P}=0.734)$ and the Egger linear regression test $(\mathrm{P}=0.547)$.

\section{DISCUSSION}

CYP2E1, a key enzyme in the metabolic activation of several carcinogens, contributes to the development of $\mathrm{CN}$. Recently, the association between CYP2E1 polymorphisms and CN risk has received increasing attention because of its potential in transcriptional regulation of gene expression (Agundez, 2004). However, in this meta-analysis, the CYP2E1 RsaI and DraI polymorphisms were not found to be correlated with $\mathrm{CN}$ risk in a codominant model (the most 
appropriate), which is consistent with the results of previous studies (Kim et al., 2000; Ferreira et al., 2006; Liu et al., 2009).

Notably, the PstI restriction site polymorphism in the 5 ' flanking region of the $C Y$ P2E1 gene has been shown to affect the transcriptional regulation of gene expression for several carcinogens (Carriere et al., 1996), which is associated with cancer development (ElZein et al., 1997; Hildesheim et al., 1997). Sierra-Torres et al. (2006) indicated that the PstI homozygous variant $(\mathrm{c} 2 / \mathrm{c} 2)$ conferred an overall greater than 6 -fold increase in the risk for high-grade squamous intraepithelial lesion in a non-Asian population, even after adjusting for wood smoke exposure and human papillomavirus-infection; however, no significant effect of the $\mathrm{c} 2 / \mathrm{c} 2$ genotype on the overall risk for CC was reported in a Korean population by Kim et al. (2000), suggesting the role of PstI in CN susceptibility may depend on ethnicities.

In this meta-analysis, we observed an association between the PstI polymorphism and $\mathrm{CN}$ risk in a recessive model (the most appropriate) with no heterogeneity, indicating that individuals with the homozygous rare genotype (c2/c2) had a higher risk of $\mathrm{CN}$ than those with the homozygous wild-type genotype (c1/c1) and heterozygous genotype (c1/c2), although statistical significance was not reached. Further results confirmed that the PstI polymorphism was significantly correlated with CN susceptibility in non-Asians. Moreover, after excluding each study, the pattern of the pooled effect size remained significant. Publication bias was not suggested in the present study, possibly because of the deliberate search strategy and data extraction methods used.

However, there were some limitations to this meta-analysis. First, the sample size was small. Second, the pooled estimates were not adjusted for confounding factors. Third, lack of the original data in the studies limited further analysis.

In conclusion, although the pooled estimates should be interpreted with caution, our meta-analysis results suggest that the CYP2E1 PstI polymorphism is associated with $\mathrm{CN}$ risk in non-Asians. However, large sample size studies in different populations, as well as more detailed data regarding individual differences and environmental factors, are warranted.

\section{Conflicts of interest}

The authors declare no conflict of interest.

\section{REFERENCES}

Agundez JA (2004). Cytochrome P450 gene polymorphism and cancer. Curr. Drug Metab. 5: 211-224.

Au WW, Sierra-Torres CH and Tyring SK (2003). Acquired and genetic susceptibility to cervical cancer. Mutat. Res. 544: 361-364.

Carriere V, Berthou F, Baird S, Belloc C, et al. (1996). Human cytochrome P450 2E1 (CYP2E1): from genotype to phenotype. Pharmacogenetics 6: 203-211.

Chen L, Shen Y, Liu L, Li X, et al. (2013). Interleukin-13 -1112 C/T promoter polymorphism confers risk for COPD: a meta-analysis. PLoS One 8: e68222.

El-Zein RA, Zwischenberger JB, Abdel-Rahman SZ, Sankar AB, et al. (1997). Polymorphism of metabolizing genes and lung cancer histology: prevalence of CYP2E1 in adenocarcinoma. Cancer Lett. 112: 71-78.

Ferreira PM, Catarino R, Pereira D, Matos A, et al. (2006). Cervical cancer and CYP2E1 polymorphisms: implications for molecular epidemiology. Eur. J. Clin. Pharmacol. 62: 15-21.

Hildesheim A, Anderson LM, Chen CJ, Cheng YJ, et al. (1997). CYP2E1 genetic polymorphisms and risk of nasopharyngeal carcinoma in Taiwan. J. Natl. Cancer Inst. 89: 1207-1212.

Kim JW, Lee CG, Park YG, Kim KS, et al. (2000). Combined analysis of germline polymorphisms of p53, GSTM1, GSTT1, CYP1A1, and CYP2E1: relation to the incidence rate of cervical carcinoma. Cancer 88: 2082-2091. 
Liu Y, Ma WJ, Liu Q, Yang T, et al. (2009). Association between genetic polymorphism of GSTM1, CYP2E1 and susceptibility to cervical cancer and its precancerous lesions in women in Xinjiang. Xian Dai Fu Chan Ke Jin Zhan 18: 840-843.

Nishino K, Sekine M, Kodama S, Sudo N, et al. (2008). Cigarette smoking and glutathione S-transferase M1 polymorphism associated with risk for uterine cervical cancer. J. Obstet. Gynaecol. Res. 34: 994-1001.

Sierra-Torres CH, Au WW, Arrastia CD, Cajas-Salazar N, et al. (2003). Polymorphisms for chemical metabolizing genes and risk for cervical neoplasia. Environ. Mol. Mutagen 41: 69-76.

Sierra-Torres CH, Arboleda-Moreno YY and Orejuela-Aristizabal L (2006). Exposure to wood smoke, HPV infection, and genetic susceptibility for cervical neoplasia among women in Colombia. Environ. Mol. Mutagen 47: 553-561.

von Keyserling H, Bergmann T, Schuetz M, Schiller U, et al. (2011). Analysis of 4 single-nucleotide polymorphisms in relation to cervical dysplasia and cancer development using a high-throughput ligation-detection reaction procedure. Int. J. Gynecol. Cancer 21: 1664-1671. 